\title{
Maximum a posteriori planet detection and characterization with a nulling interferometer
}

\author{
Eric Thiébaut ${ }^{1}$ and Laurent Mugnier ${ }^{2}$ \\ ${ }^{1}$ Jean-Marie Mariotti Center/Centre de Recherches Astronomiques de Lyon \\ 9, avenue Charles André; F-69561 Saint Genis Laval Cedex, France \\ email: thiebaut@obs.univ-lyon1.fr \\ ${ }^{2}$ Jean-Marie Mariotti Center/Office National d'Etudes et de Recherches Aérospatiales \\ B.P. 72, 92322 Châtillon Cedex, France \\ email: Laurent.Mugnier@onera.fr
}

\begin{abstract}
Nulling interferometers such as Darwin or TPF will require a rather sophisticated data processing in order to perform a reliable planet detection and characterization. We propose a Bayesian method, which follows the maximum a posteriori (MAP) approach, to solve this problem. Our method accounts for the noise statistics and optimally combines the data from a nulling interferometer at all observed wavelengths to perform reliable planet detection. The problem to be solved is however multi-modal. We show how, in practice, the global optimum of the MAP criterion can be found by our method; the latter also provides the most likely spectral energy distributions of all planets. Additionally, we show that a proper regularization allows us to achieve an improved robustness of the detection and could lead to shorter observation times.
\end{abstract}

Keywords. techniques: interferometric, methods: data analysis, methods: numerical, methods: statistical, planetary systems.

\section{Introduction}

Nulling interferometers make use of destructive interferences to get rid of the stellar light on the line of sight and strongly reduce the contrast between the light received from a star and from its surrounding planets Bracewell (1978). It is expected that this feature, possibly combined with internal and external modulations, will allow us to detect direct evidences of exo-solar planet emission. A nulling interferometer however gives data of very low signal-to-noise ratio and which are far from a direct image of the observed scene. The planet detection and their characterization by their spectral signatures therefore require a tailor-made data processing. In this paper, we propose a new method based on the maximum a posteriori (MAP) approach, in order to achieve an optimal planet detection and characterization. First, the assumed data model for a nulling interferometer is established. Then we explain how the MAP approach can be applied to this kind of data. We also show how to effectively obtain the globally optimal solution: that is the most likely planet positions and spectral energy distributions. Finally, we demonstrate the capabilities of our algorithm by the processing of realistic simulated data. 


\section{Data Model}

A general model of the output from a nulling interferometer writes Mennesson (1999):

$$
A_{t, \lambda}=\iint R_{t, \lambda}(\boldsymbol{\theta}) I_{\lambda}(\boldsymbol{\theta}) \mathrm{d} \boldsymbol{\theta},
$$

where $A_{t, \lambda}$ is the recorded amplitude at time $t$ and for the effective wavelength $\lambda, R_{t, \lambda}(\boldsymbol{\theta})$ is the response of the instrument, i.e., the transmission map as a function of angular coordinates $\boldsymbol{\theta}$, and $I_{\lambda}(\boldsymbol{\theta})$ is the specific brightness distribution of the observed object (or scene). In this model, we assume that the scene does not vary during the whole data acquisition. Doing otherwise would make the inversion prohibitively complicated and probably not robust at least for the detection stage.

Spatial nulling interferometers such as Darwin will use both external modulation (i.e., rotation of the interferometric array with time) and internal modulation (i.e., modification of the transmission map via phase shifts in the beam combination), so one should bear in mind that index $t$ in Eq. (2.1) actually codes for both the position in time and for the configuration of the phase shifts of the array. Historically, the concept of internal modulation was imagined by Mariotti in 1997 Mennesson, Léger \& Ollivier (2005).

Because the data model of Eq. (2.1) is linear, any linear combination of the data gives synthetic data that follow the same model, with a synthetic transmission map that is the corresponding linear combination of transmission maps. When using internal modulation it is possible to find coefficients for such a linear combination that make the synthetic transmission map an odd function with respect to the angular direction $\boldsymbol{\theta}$ Absil (2001). Additionally, it is trivial to show from Eq. (2.1) that the data corresponding to an odd $R$ and an even object's brightness distribution is zero. This is of paramount importance as all components of the observed scenes except the planets should be even within a good approximation. Indeed, the star and the exo-zodiacal light should be symmetrical, and the local zodiacal dust as well as the instrumental thermal emissions are at a constant level across the field of view. This means that we can, in our data model, retain only the contribution of the planets, and search only for their parameters in the inversion. We can write the specific object's brightness distribution as:

$$
I_{\lambda}(\boldsymbol{\theta})=\sum_{i=1}^{N_{\mathrm{src}}} F_{i, \lambda} \delta\left(\boldsymbol{\theta}-\boldsymbol{\theta}_{i}\right)+I_{\lambda}^{\mathrm{sym}}(\boldsymbol{\theta}),
$$

where $N_{\text {src }}$ is the number of planets and $F_{i, \lambda}$ is the spectral energy distribution (SED) of the $i$-th planet and $I_{\lambda}^{\mathrm{sym}}(\boldsymbol{\theta})$ accounts for all the symmetrically distributed sources. After proper linear combination of the data, $I_{\lambda}^{\text {sym }}(\boldsymbol{\theta})$ does not contribute to the expected value of the signal; it is however the main source of noise. Combining the data model of Eq. (2.1) with the object's model of Eq. (2.2) and accounting for modulation yields:

$$
A_{t, \lambda}=\sum_{i=1}^{N_{\mathrm{src}}} R_{t, \lambda}\left(\boldsymbol{\theta}_{i}\right) F_{i, \lambda}+n_{t, \lambda},
$$

where $n_{t, \lambda}$ is the detection noise.

\section{Detection Algorithm}

The problem at hand is to estimate the positions $\boldsymbol{\theta}_{i}$ and the SED's $F_{i, \lambda}$ of the planets, assuming that their number $N_{\mathrm{src}}$ is known. Let us denote this set of parameters by $(\boldsymbol{\theta}, \mathbf{F}) \triangleq\left\{\boldsymbol{\theta}_{i}, F_{i, \lambda} ; i=1, \ldots, N_{\mathrm{src}} ; \lambda_{\min } \leqslant \lambda \leqslant \lambda_{\max }\right\}$. 
A well-known approach for the detection task is correlation. One could for instance consider correlating the data recorded at a given wavelength with the noiseless data model obtained for a point-source at all possible locations Angel \& Woolf (1997), Mennesson \& Mariotti (1997). The location yielding the highest correlation would be a probable position for a planet. Yet, let alone the fact that such a scheme is not statistically appropriate for an instrument that is not shift-invariant, it neither makes use of statistical information on the noise, nor provide a way to optimally combine the data at several wavelengths.

The maximum a posteriori (MAP) approach has none of these shortcomings; it defines the solution $(\hat{\boldsymbol{\theta}}, \hat{\mathbf{F}})$ as the set of parameters that has maximum probability given the data:

$$
(\hat{\boldsymbol{\theta}}, \hat{\mathbf{F}})=\arg \max _{(\boldsymbol{\theta}, \mathbf{F})} \operatorname{Pr}(\boldsymbol{\theta}, \mathbf{F} \mid \mathbf{A}),
$$

where A denotes the data set. By Bayes' theorem and since the probability $\operatorname{Pr}(\mathbf{A})$ of the data alone does not depend on the sought parameters, the MAP solution also solves:

$$
(\hat{\boldsymbol{\theta}}, \hat{\mathbf{F}})=\arg \max _{(\boldsymbol{\theta}, \mathbf{F})} \operatorname{Pr}(\mathbf{A} \mid \boldsymbol{\theta}, \mathbf{F}) \operatorname{Pr}(\boldsymbol{\theta}, \mathbf{F})=\arg \min _{(\boldsymbol{\theta}, \mathbf{F})} J_{\mathrm{MAP}}(\boldsymbol{\theta}, \mathbf{F}),
$$

where the so-called maximum a posteriori cost function (or criterion) is:

$$
J_{\text {MAP }}(\boldsymbol{\theta}, \mathbf{F})=J_{\text {data }}(\boldsymbol{\theta}, \mathbf{F})+J_{\text {prior }}(\boldsymbol{\theta}, \mathbf{F}),
$$

and where $J_{\text {data }}(\boldsymbol{\theta}, \mathbf{F}) \propto-\log \operatorname{Pr}(\mathbf{A} \mid \boldsymbol{\theta}, \mathbf{F})$ is the neg-log-likelihood of the data given the model and $J_{\text {prior }}(\boldsymbol{\theta}, \mathbf{F}) \propto-\log \operatorname{Pr}(\boldsymbol{\theta}, \mathbf{F})$ is the negative logarithm of the a priori probability of the model. Assuming independent Gaussian noise, $J_{\text {data }}$ writes:

$$
J_{\mathrm{data}}(\boldsymbol{\theta}, \mathbf{F}) \triangleq \sum_{t, \lambda} \frac{1}{\sigma_{t, \lambda}^{2}}\left(A_{t, \lambda}-\sum_{i=1}^{N_{\mathrm{src}}} R_{t, \lambda}\left(\boldsymbol{\theta}_{i}\right) F_{i, \lambda}\right)^{2}
$$

where the noise variance $\sigma_{t, \lambda}^{2}$ can be estimated from the data. Minimizing the term $J_{\text {data }}$ enforces agreement of the parameters with the data; and minimizing $J_{\text {prior }}$ enforces agreement of the parameters with the available a priori information. In inverse problem jargon, $J_{\text {prior }}$ is called a regularization term. Since a properly sampled planet spectrum should be a smooth function of the wavelength, we define $J_{\text {prior }}$ so as to estimate the roughness of the SED's:

$$
J_{\text {prior }}(\boldsymbol{\theta}, \mathbf{F}) \triangleq \sum_{i=1}^{N_{\mathrm{src}}} \mu_{i} \sum_{\lambda=\lambda_{\min }}^{\lambda_{\max }}\left(\frac{\partial^{m} F_{i, \lambda}}{\partial \lambda^{m}}\right)^{2}
$$

where the $m$ th-order spectral derivative is computed by finite differences and were $\mu_{i}$ are hyper-parameters allowing us to tune the relative weight of regularization.

Finding the minimum of $J_{\text {MAP }}$ with respect to $(\boldsymbol{\theta}, \mathbf{F})$ yields the optimal solution. However, such a criterion is multi-modal, hence its deepest minimum cannot be found by a descent optimization algorithm: global optimization is required. Nevertheless, since $J_{\text {data }}$ and $J_{\text {prior }}$ are convex with respect to the subset of parameters $\mathbf{F}$, the most likely SED's can be obtained by solving:

$$
\frac{\partial J_{\mathrm{MAP}}(\boldsymbol{\theta}, \mathbf{F})}{\partial F_{i, \lambda}}=0 ; \forall i, \forall \lambda,
$$

for given planet positions. Moreover, because both criteria $J_{\text {data }}$ of Eq. (3.4) and $J_{\text {prior of }}$ Eq. (3.5) are quadratic with respect to the SED's, JMAP is also quadratic and Eq. (3.6) is a set of linear equations. Its solution, denoted in the following by $\hat{\mathbf{F}}(\boldsymbol{\theta})$, is analytical and depends on the data and on the considered planet positions. 


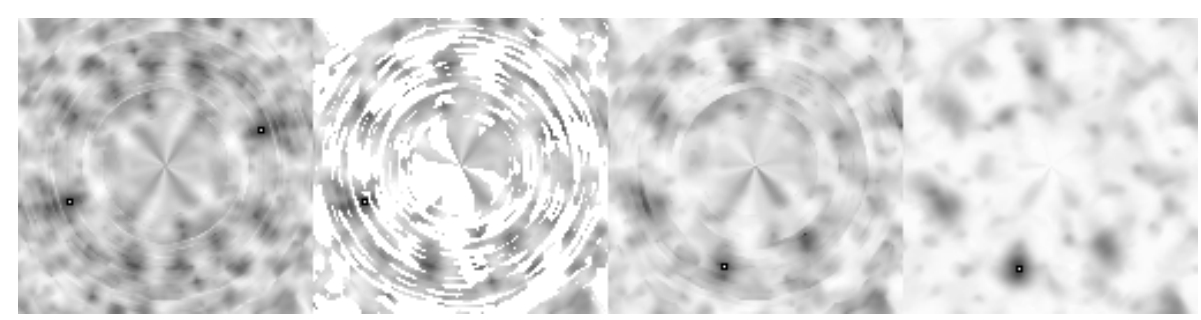

Figure 1. Detection of a faint planet by various criteria. From left to right: unconstrained $J_{\text {data }}^{\dagger}$, $J_{\text {data }}^{\dagger}$ with negative SED's removed, $J_{\text {data }}^{\dagger}$ with positivity and, $J_{\text {MAP }}^{\dagger}$ with positivity. Darkest values indicate most likely planet positions. The global minima of the criteria are highlighted as a white dot surrounded by a black border.

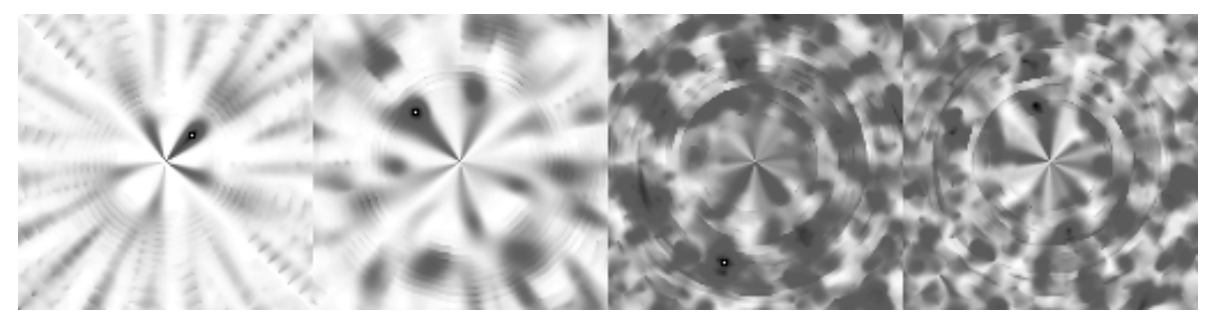

Figure 2. Multiple planet detection steps at low signal-to-noise ratio $(\mathrm{RSB} \sim 2)$. From left to right: criterion mapped for the 1st, 2 nd, 3rd and 4th candidate planet.

If we replace the SED's by $\hat{\mathbf{F}}(\boldsymbol{\theta})$ in the cost function $J_{\mathrm{MAP}}(\boldsymbol{\theta}, \mathbf{F})$ of Eq. (3.2) we obtain a partially optimized cost function with many less parameters, which only depends (explicitly) on the assumed planet positions:

$$
J_{\mathrm{MAP}}^{\dagger}(\boldsymbol{\theta}) \triangleq J_{\mathrm{MAP}}(\boldsymbol{\theta}, \hat{\mathbf{F}}(\boldsymbol{\theta})) \text {. }
$$

It must be noted that this new cost function $J_{\text {MAP }}^{\dagger}(\boldsymbol{\theta})$ is nothing but the original MAP cost function $J_{\mathrm{MAP}}(\boldsymbol{\theta}, \mathbf{F})$ that has been optimized on a subset of its parameters (namely, on the SED's). Consequently, the former has exactly the same minima as the latter and is significantly simpler to optimize.

Simply by setting $\mu_{i}=0, \forall i$, it is possible to turn regularization off to obtain a solution which does not depend on any prior. In this case, $J_{\mathrm{MAP}}=J_{\text {data }}$ and hence $J_{\text {MAP }}^{\dagger}=J_{\text {data }}^{\dagger}$. To demonstrate the improvement brought by regularization, we must compare the results obtained by optimizing the unregularized criterion $J_{\text {data }}^{\dagger}$ and those obtained with $J_{\text {MAP }}^{\dagger}$. In the following and for sake of simplicity, we denote by $J(\boldsymbol{\theta}, \mathbf{F})$ the actual criterion (either $J_{\text {MAP }}$ or $J_{\text {data }}$ ) and $J^{\dagger}(\boldsymbol{\theta})$ the corresponding partially optimized criterion.

The minimum of $J^{\dagger}$ provides the most likely set of planet positions. As expected and shown by Fig. $1, J^{\dagger}$ is still multi-modal with respect to $\boldsymbol{\theta}$. To find the global minimum of $J^{\dagger}$, the most obvious approach is to sample the field of view and map the cost function $J^{\dagger}$ onto the grid of planet positions. However if the grid has $N_{\text {grid }}$ cells, this requires the solving of Eq. (3.6) and the computation of $J^{\dagger}$ for $N_{\text {grid }}{ }^{N_{\text {src }}}$ cases. Such a global search is therefore limited to a modest number of planets on a reasonably small grid. In the single planet case (see Fig. 1), $J^{\dagger}$ is a 2-D pseudo-image of the field of view where local minima corresponds to the most likely locations of the planet.

Obviously, the first pseudo-image shown by Fig. 1 is even, which constitutes a sign ambiguity on the planet position. This ambiguity is due to the fact that the considered synthetic transmission map $R$ is odd so that for any $(\boldsymbol{\theta}, \mathbf{F}),(-\boldsymbol{\theta},-\mathbf{F})$ has exactly the same likelihood. Simply selecting the SED that is mostly positive removes the ambiguity. The 

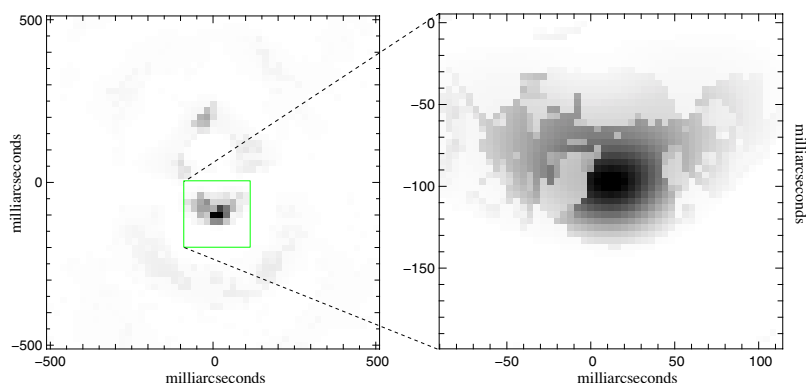

Figure 3. Planet detection map for the blind-test. The darkest parts of the maps indicate the minima of the criterion, hence where the planet is most likely located.

fact that the SED of each planet must actually be positive at all wavelengths suggests an enhancement to the SED estimation, which consists in minimizing Eq. (3.2) or Eq. (3.4) with respect to the SED's under a positivity constraint:

$$
\hat{\mathbf{F}}(\boldsymbol{\theta})=\arg \min _{\mathbf{F}} J(\boldsymbol{\theta}, \mathbf{F}) \quad \text { s.t. } \quad F_{i, \lambda} \geqslant 0 ; \forall i, \forall \lambda .
$$

The solution is no longer analytical but improves the planet detection, as seen by the second and third pseudo-images in Fig. 1. Many numerical algorithms have been devised to solve Eq. (3.8) when $J$ is quadratic Lawson \& Hanson (1974), Bro \& Jong (1997), Stark \& Parker (1995). In our simulations, we solved Eq. (3.8) with VMLM-B Thiébaut (2002), which is suitable even for non-quadratic criteria.

\section{Simulation Results}

We first checked our algorithm with a scene made of a central star, an exo-zodiacal cloud and 1 or 3 planets. We simulated data for 4 different rotation angles of a RobinLaurance interferometer with 15 spectral channels. In the case of a single planet, Fig. 1 clearly demonstrates the improvements brought by non-negativity and regularization: not only do these constraints help the method remove ambiguities (there are fewer local minima of similar depth in the criterion map) but they also yield the correct position, which would have been completely wrong otherwise. In order to be able to detect more than one planet we iteratively applied our single planet detection scheme to a 3 planet scene as follows: for each new planet, we optimize $J^{\dagger}$ with respect to all SED's but we only allow the new planet to move; this yields a 2-D pseudo-image, whose global minimum is the most likely position of the new planet. Figure 2 shows that the 3 first planets are readily detected by our algorithm, whereas the (non-existing) 4th one is not.

In order to test our algorithm when the components of the observed scene are really unknown, we applied our method to nulling data simulated by someone else. The data consists in 1000 exposures of a rotating linear nulling interferometer with 3 telescopes and 2 internal modulation states (the so-called L-TTN configuration). There are 27 wavelength channels from $6.7 \mu \mathrm{m}$ to $20 \mu \mathrm{m}$, the duration of each exposure is $100 \mathrm{~s}$ and we estimated the signal-to-noise ratio to be $\mathrm{SNR} \simeq 1.5$ per datum. Figure 3 shows the regularized criterion $J_{\text {MAP }}^{\dagger}$ computed for this data set onto a $1^{\prime \prime} \times 1^{\prime \prime}$ field of view around the central star. It clearly exhibits a likely point-like source at $\left(0{ }^{\prime \prime} 012,-00^{\prime \prime} 097\right)$ from the central star. After unveiling of the simulation parameters, a planet was indeed simulated at $1 \mathrm{AU}$ away from a star at a distance of $10 \mathrm{pc}$. To check the robustness of our algorithm, we repeated the processing for smaller and smaller data subsets. Figure 4 shows the result of the detection with $100 \%, 50 \%, 10 \%, 5 \%$ and $1 \%$ of the original data, i.e., with 


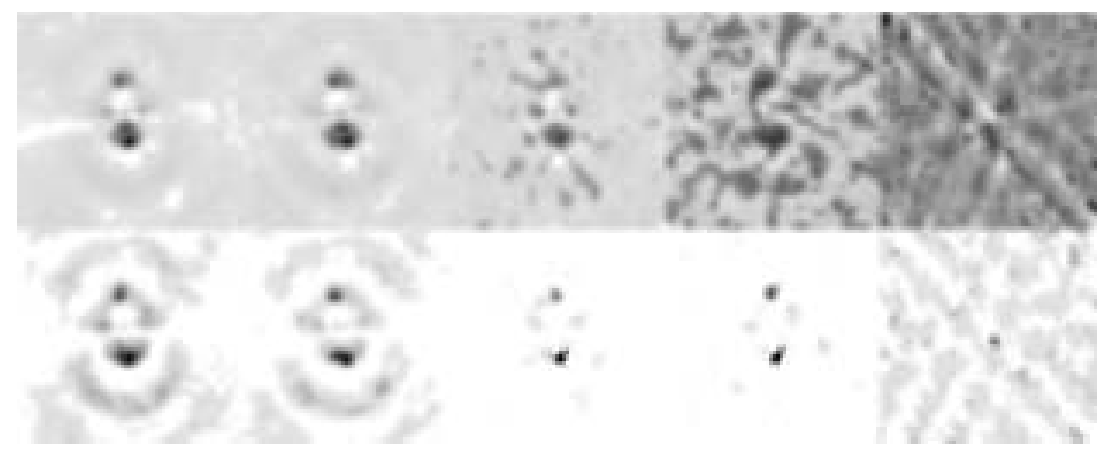

Figure 4. Effects of reducing the total observing time on planet detection. Top row: planet detection by unregularized criterion $J_{\text {data }}^{\dagger}$. Bottom row: planet detection with spectral regularization criterion $J_{\text {MAP }}^{\dagger}$. From left to right: 1000, 500, 100, 50 and 10 exposures. Strict positivity constraint was used in all cases.

$1000,500,100,50$ and only 10 exposures of $100 \mathrm{~s}$ each. From this figure it is clear that spectral regularization improves the sensitivity of the detection. Indeed, with spectral regularization, the detection is still reliable with only $\sim 50$ exposures; whereas at least $\sim 100$ exposures are needed without the regularization.

\section{Conclusions}

We have shown how a maximum a posteriori approach can lead to a planet detection algorithm suitable for nulling interferometry data. Unlike the correlation method used in earlier studies, our approach optimally combines all the available pieces of information. Thanks to simulated data, we have demonstrated that non-negativity and spectral regularization (enforcement that the spectral energy distribution of a planet should be somewhat smooth) are critical features to disentangle the numerous ambiguities in the detection from such kind of data. The most likely SED's of the detected planets are a by-product of our algorithm and can be used to perform planet characterization. We have also shown how the detection scheme can be modified to account for several planets.

\section{Acknowledgements}

This work has been supported by ESA under contract Reconstruction of Exo Solar System Properties (RESSP). The scene for our multiple planet detection tests was computed by A. Belu (LUAN). The data for our blind-test were kindly provided by R. den Hartog (ESA).

\section{References}

Absil, O. 2001, PhD thesis, Université de Liêge, Belgique Angel, J. R. P. \& Woolf, N. J. 1997, Ap. J. 475, 373-379 Bracewell, R. N. 1978, Nature 274, 780-781

Bro, R. \& Jong, S.D. 1997, Journal of Chemometrics 11, 393-401

Lawson, C. L. \& Hanson, R. J. 1974, Solving Least Squares Problems, Prentice-Hall

Mennesson, B. \& Mariotti, J.-M. 1997, Icarus 128, 202-212

Mennesson, B. 1999, PhD thesis, Université de Paris VII, France

Mennesson, B., Léger, A. \& Ollivier, M. 2005, Icarus 178, 570-588

Stark P.B. \& Parker R.L. 1995, Comp. Stat. 10, 129-141

Thiébaut, E. 2002, in Proc. SPIE 4847, 174-183 